\title{
On the Sensing Mechanisms of a Hydroresistive Flexible Film based on an Organic Molecular Metal
}

Raphael Pfattner, ${ }^{1,2^{*}}$ Elena Laukhina, ${ }^{2, *}$ Laura Ferlauto, ${ }^{3, \dagger}$ Fabiola Liscio, ${ }^{3}$ Silvia Milita, ${ }^{3}$ Anna Crespi, ${ }^{1}$ Victor Lebedev, ${ }^{1,2,1}$ Marta Mas-Torrent, ${ }^{1,2}$ Vladimir Laukhin, ${ }^{1,2,4}$ Concepció Rovira ${ }^{1,2}$ and Jaume Veciana ${ }^{1,2, *}$

1) Institut de Ciència de Materials de Barcelona (ICMAB-CSIC) Campus UAB, 08193 Bellaterra, Spain

2) Networking Research Center on Bioengineering Biomaterials and Nanomedicine (CIBER-BBN) Campus UAB, 08193 Bellaterra, Spain

3) Consiglio Nazionale delle Ricerche (CNR)-Instituto per la Microelettronica e Microsistemi (IMM), 40129 Bologna, Italy

4) Institució Catalana de Recerca i Estudis Avançats (ICREA), 08193-Bellaterra, Spain.

$\dagger$ †) Current Address: EPFL STI IBI-STI LNE Campus Biotech, CH-1202 Geneva, Switzerland

॥) Current Address: Dow Europe GmbH, CH-8810 Horgen, Switzerland

Corresponding Authors

Dr. Raphael Pfattner*, Email address: rpfattner@icmab.es

Dr. Elena Laukhina*, Email address: laukhina@icmab.es

Prof. Jaume Veciana*, Email address: vecianaj@icmab.es. 
Table of Contents:

$\begin{array}{lr}\text { S1) Preparation of BL-films } & \text { S3 }\end{array}$

S2) X-Ray diffraction pattern of BL-films $\quad$ S4

S3) Electromechanical characterization of BL-films $\quad$ S7

S4) Hydro-sensitivity of BL-Films F1 S10

S5) Boltzmann's sigmoidal equation and de-convolution of $\mathbf{R} / \mathbf{R}_{\mathbf{0}}$ change $\quad$ S20 


\section{S1) Preparation of BL films}

The preparation of BL films consist of a simple two step procedure shown schematically in Figure S1. This procedure leads to a polycrystalline highly conducting topmost layer (BEDT$\mathrm{TTF}_{\mathrm{x}} \mathrm{Hal}_{\mathrm{y}}$ ) of about $\mathrm{d} \sim 2 \mu \mathrm{m}$ thickness grown on top of $18-23 \mu \mathrm{m}$ thick polymeric (PC) support.

First step: Composite film preparation

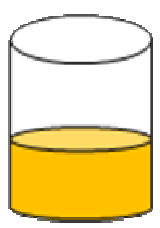

1,2 - dichlorobenzene solution of: 98 wt. $\%$ (PC) + 2 wt. \% (BEDT-TTF)

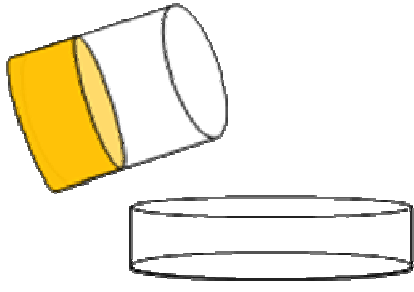

Film casting at $130^{\circ} \mathrm{C}$

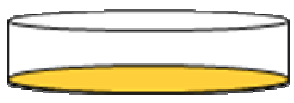

Composite film of 20-25 $\boldsymbol{\mu m}$ thickness: 98 wt.\% (PC) + 2 wt.\% (BEDT-TTF)

Second step: preparation of conductive covering layer of molecular conductor: (BEDT-TTF) ${ }_{\mathrm{x}} \mathrm{Hal}_{\mathrm{y}}$
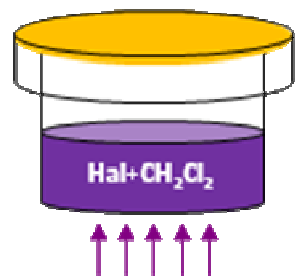

Treatment of the film surface with vapors of $\mathrm{I}_{2}$ or $(\mathrm{Br}) / \mathrm{CH}_{2} \mathrm{Cl}_{2}$ inducing a reaction: BEDT-TTF"+H al $\rightarrow$ BEEDT-TTF: Hal

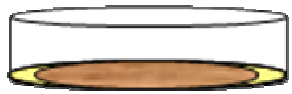

High esndurti:? BLfillT

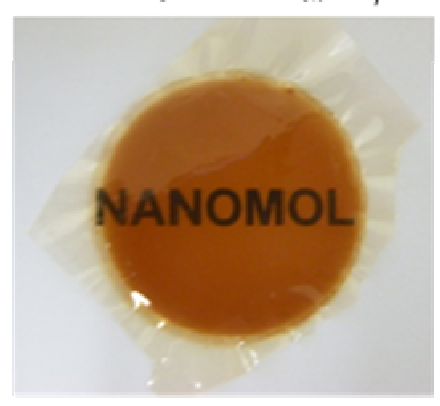

Fhoto of a BL film; bro:? nush areas

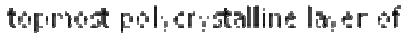

(BEDT-TTF) ${ }_{2} \mathrm{Hal}_{\text {, }}$

Figure S1. Two step preparation of BL-films. Top) First step consisting of casting a hot solution of PC (98 wt.\%) and BEDT-TTF ( $2 \mathrm{wt} . \%)$ in $1,2-$ dichlorobenzene (conc. $=20 \mathrm{~g} / \mathrm{l}$ ) into a Petri dish at $\mathrm{T}=130{ }^{\circ} \mathrm{C}$ and evaporation of solvent leading to a composite film with thickness of about $20-25 \mu \mathrm{m}$. Bottom) Second step: film treatment with halogen vapors and formation of polycrystalline conducting layer with thickness of about $\mathrm{d} \sim 2 \mu \mathrm{m}$. 


\section{S2) X-Ray diffraction pattern of BL-films}

XRD spectra of F2 and F3 measured on a modular X-ray diffractometer RIGAKU with monochromatic $\mathrm{Cu}-\mathrm{K} \alpha(\lambda=1.54056 \AA)$ at $\mathrm{RH}=45 \pm 5 \%$ and $\mathrm{T}=24 \pm 3{ }^{\circ} \mathrm{C} .{ }^{1}$

(a)

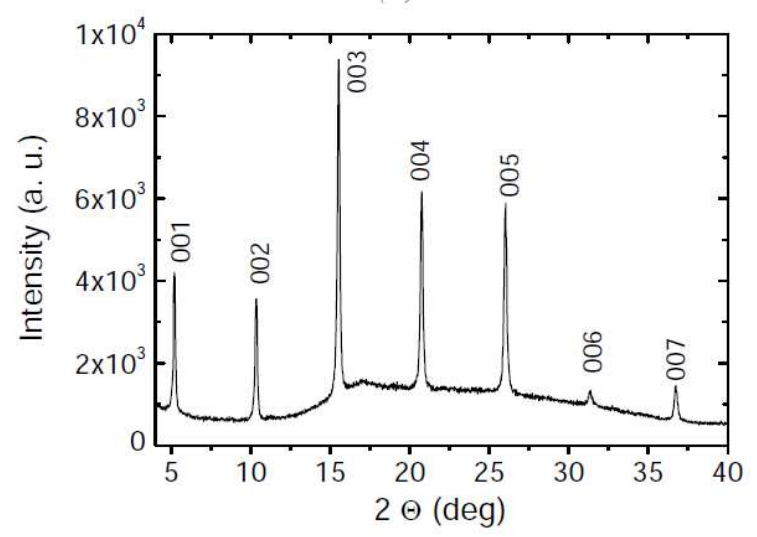

(b)

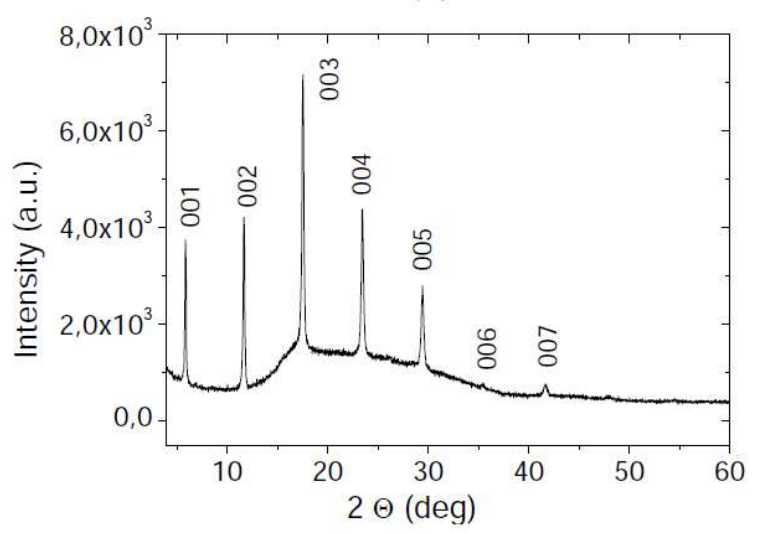

Figure S2. X-Ray diffraction pattern of BL-Films. a) F2 and b) F3. ${ }^{1}$

Figure S3 shows XRD spectrum of F1 measured on a modular X-ray diffractometer RIGAKU with monochromatic $\mathrm{Cu}-\mathrm{K} \alpha(\lambda=1.54056 \AA)$ at $\mathrm{RH}=45 \pm 5 \%$ and $\mathrm{T}=24 \pm 3{ }^{\circ} \mathrm{C}$. Two group of reflections in agreement with literature values have been found: 0k0 of phase-I in green, ${ }^{2}$ and h00 of phase II in red $^{3}$. The third group of reflections found in F1 (blue) could be clearly identified as crystals of the neutral donor molecule and stays well in agreement with the $0 \mathrm{kl}=011$ reflections of the crystal structure reported by Kobayashi et al. ${ }^{4}$ See also Table S1. This also stays in agreement with isolated, neutral donor molecules that are not participating in electrical charge transport, and are therefore not responsible for the hydro-sensitivity of F1. 

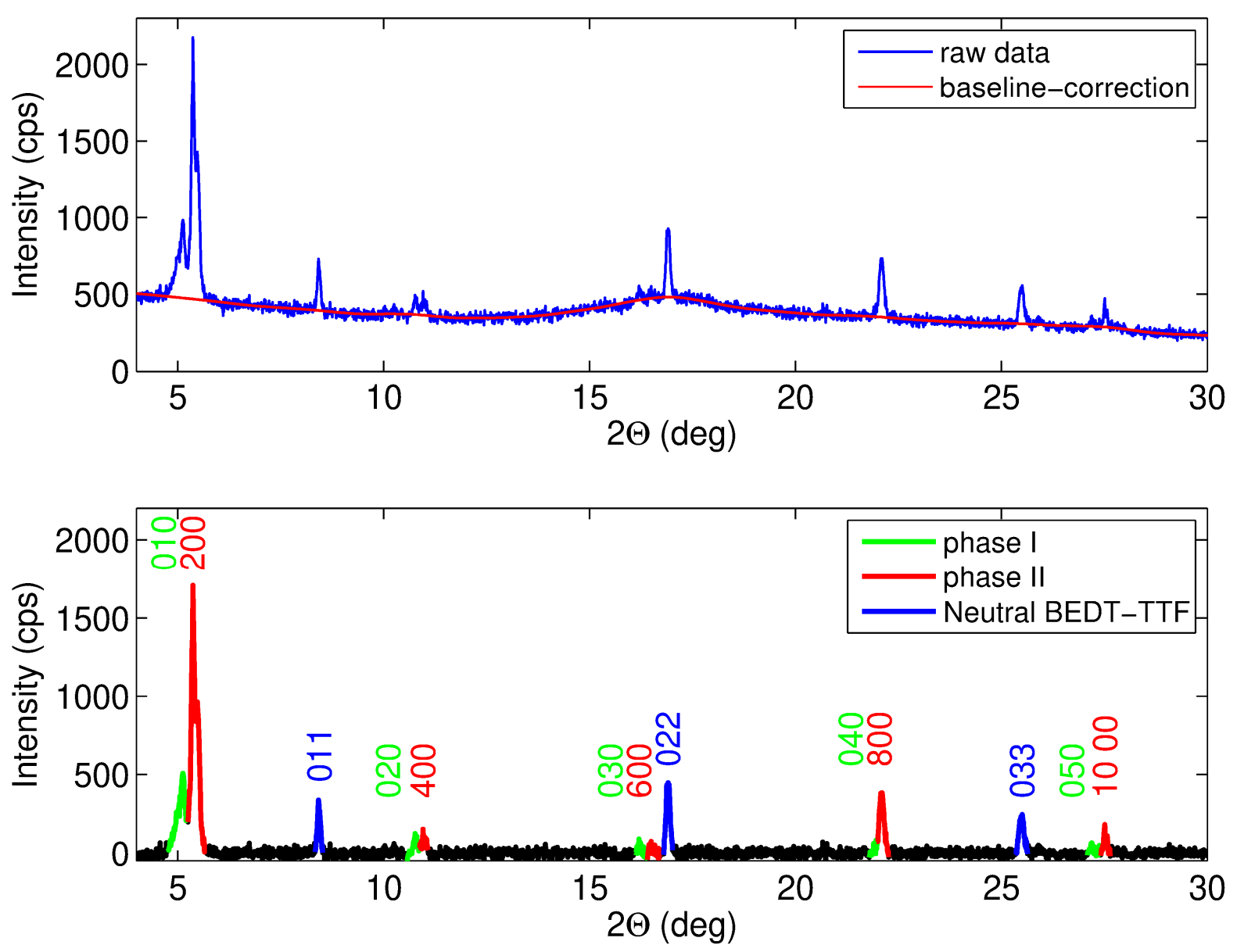

Figure S3. X-Ray diffraction pattern of BL-Film F1. Top) Raw data with baseline correction. Bottom) baseline corrected diffraction pattern with three groups of reflections orientated perpendicular to BL-film surface: 0k0 of phase-I in green, ${ }^{2}$ h00 of phase-II in red ${ }^{3}$ and $0 \mathrm{kl}$ of neutral donor molecule BEDT-TTF single crystals in blue. ${ }^{4}$ 
Table S1: X-Ray structural parameters of BL-Film F1.

\section{Neutral BEDT-TTF}

\begin{tabular}{|c|c|c|}
\hline hkl & two-theta $^{\text {a }}$ & two-theta $^{\text {b }}$ \\
\hline 011 & 8.46 & 8.42 \\
\hline 022 & 16.97 & 16.91 \\
\hline 033 & 25.58 & 25.51 \\
\hline
\end{tabular}

a) Values extracted using Mercury from CIF file of ref ${ }^{4}$

b) Values extracted from X-ray diffraction pattern of BL-film F1 of this work. 


\section{S3) Electromechanical characterization}

a)

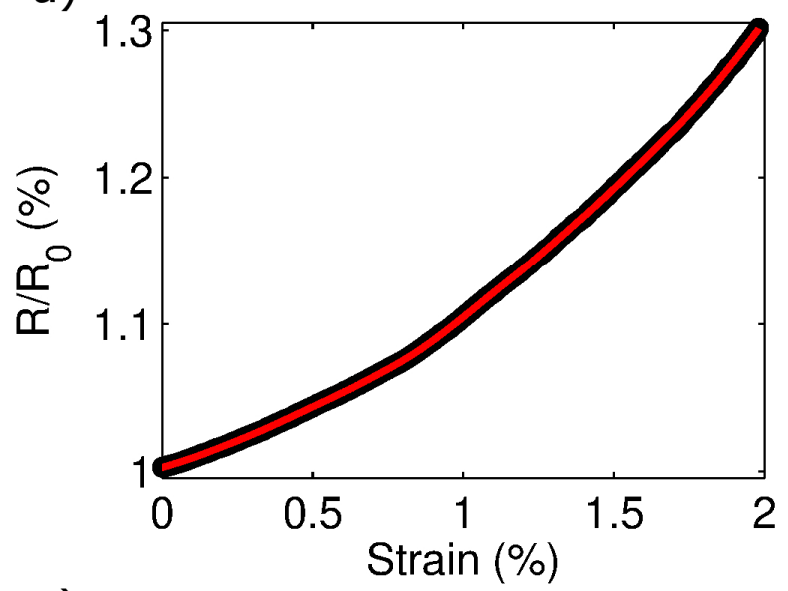

c)

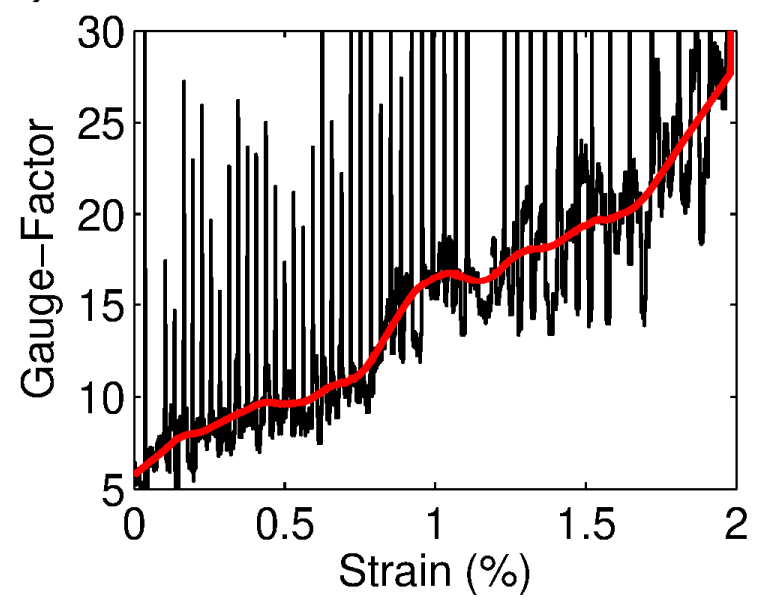

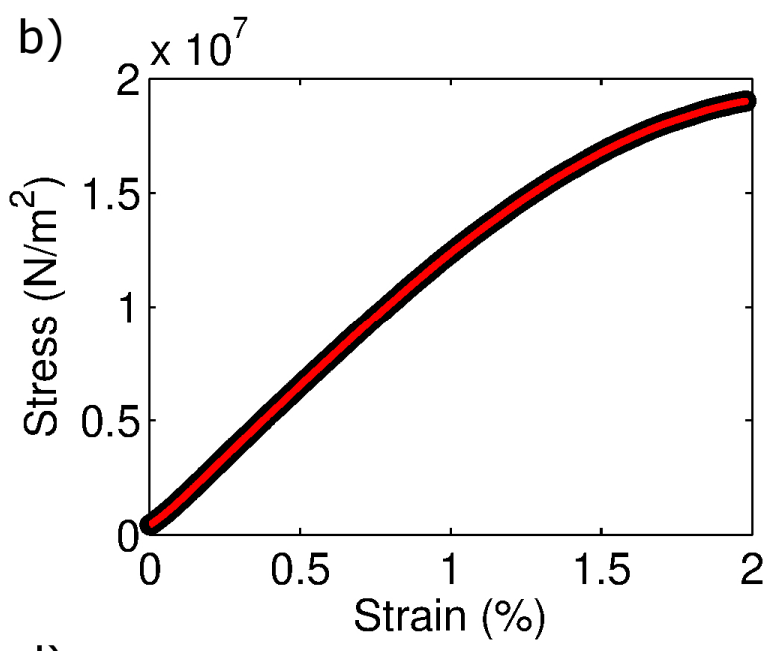

d)

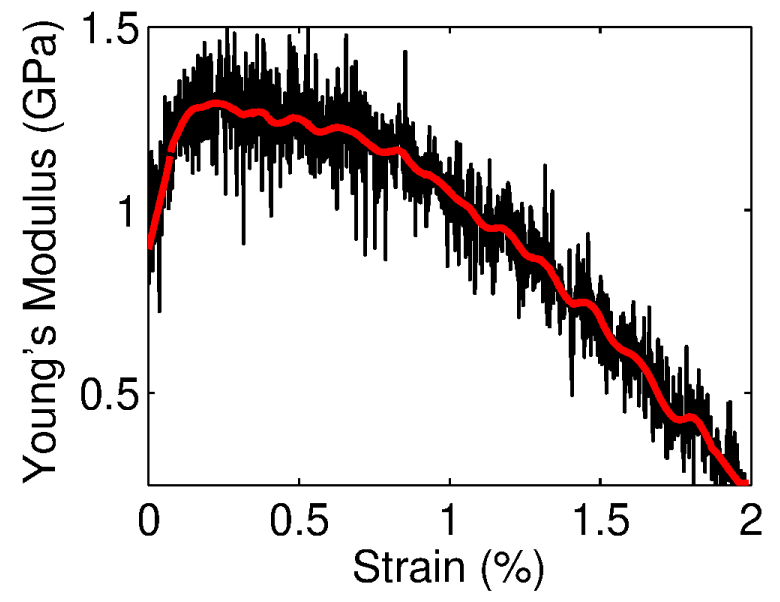

Figure S4. Electromechanical characterization of F1. a) Relative resistance versus strain and b) stress versus strain for values up to $\varepsilon=2 \%$. c) Generalized gauge-factor and d) Young's Modulus extracted for the full range. Black lines correspond to measured data which include noise originating from mechanical movement of the stretching station (i.e. stepwise deformation). Red traced are noise corrected data and calculations. 
Figure S5 shows the elastic deformation range for strain values $\varepsilon<0.6 \%$. In agreement with previous measurements, both gauge factor and Young's Modulus are fairly constant inside this range exhibiting $\mathrm{k}=9 \pm 1$, and $\mathrm{YM}=1.27 \pm 0.03 \mathrm{GPa} .{ }^{5}$ Peaks observable in the gauge factor (calculated as derivative of $\mathrm{R} / \mathrm{R}_{0}$ with respect to strain) originate from non-fully-linear and continuous deformation of stretching system and reflect once more the high sensitivity of BLfilms for strain gauges.
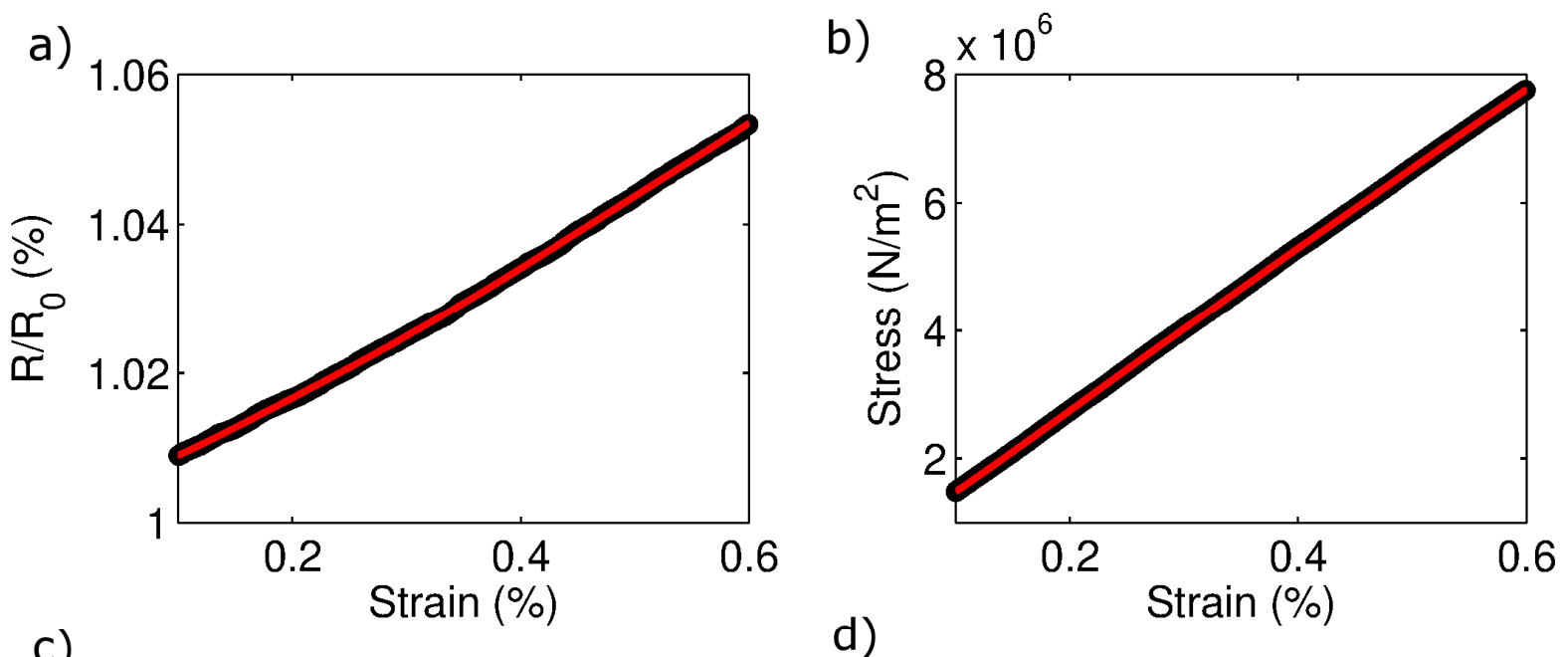

c)
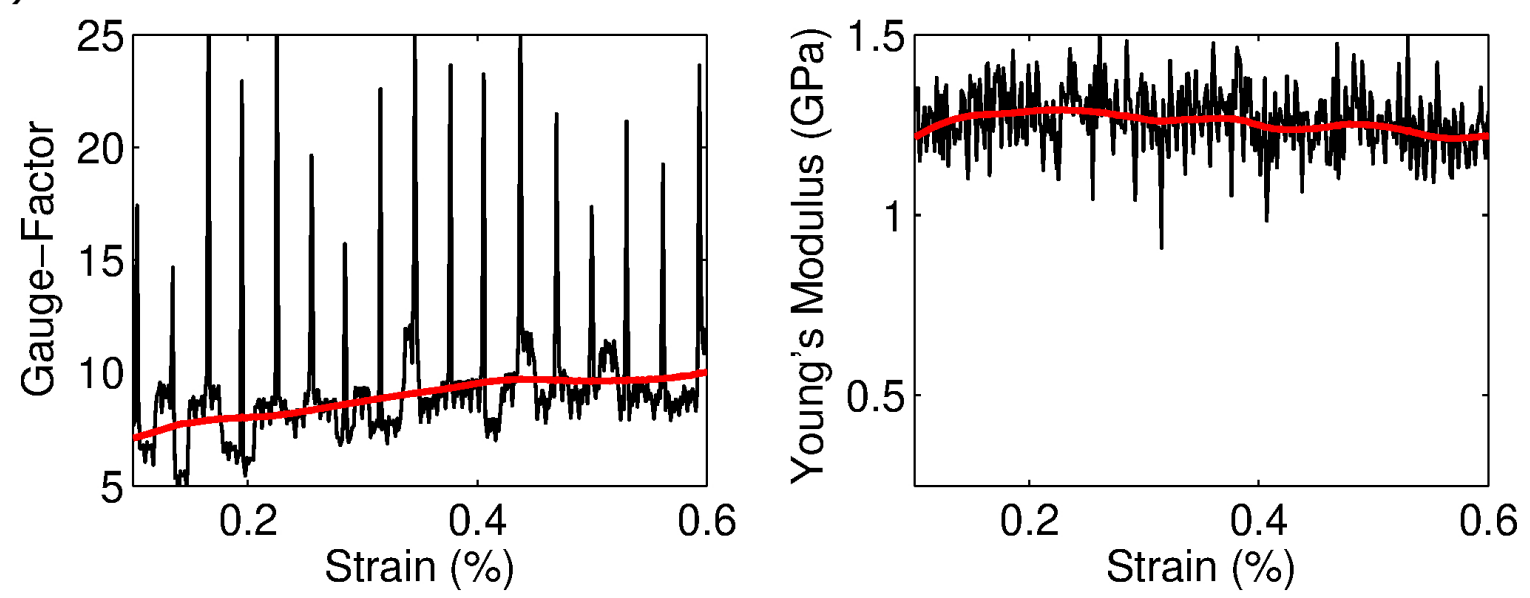

Figure S5. Electromechanical characterization of F1 - linear elastic regime. a) Relative resistance versus strain and b) stress versus strain for values up to $\varepsilon=0.6 \%$, in the linear reversible elastic range of deformation. c) gauge factor and d) Young's Modulus extracted for $\varepsilon$ $<0.6 \%$. Black lines correspond to measured data which include noise originating from mechanical movement of the stretching station (i.e. step-wise deformation). Red traced are noise corrected data and calculations. 
Table S2. Summary of performed monoaxial deformation studies for BL-films F2 and F3. $\Delta \mathrm{L}=$ elongation and $\mathrm{k}=$ gauge factor. F2: $\mathrm{L}_{0}=4.15 \mathrm{~mm}, \mathbf{F 3}: \mathrm{L}_{0}=4.0 \mathrm{~mm}$. Arithmetic mean values calculated assuming Gaussian distributed data. Table data correspond to experiments carried out in. ${ }^{5}$

\begin{tabular}{|c|c|c|c|c|}
\hline BL-Film & IRS & $\Delta \mathbf{L}[\boldsymbol{\mu m}]$ & $\varepsilon[\%]$ & $\mathbf{k}$ \\
\hline \multirow[t]{2}{*}{$\mathbf{F} 2$} & $\alpha-[\text { BEDT-TTF }]_{2} \mathrm{I}_{3}$ & $\begin{array}{l}11 \\
20 \\
30 \\
22 \\
25\end{array}$ & $\begin{array}{l}0.26 \\
0.48 \\
0.72 \\
0.53 \\
0.60\end{array}$ & $\begin{array}{c}9.66 \\
10.10 \\
9.66 \\
9.94 \\
9.95\end{array}$ \\
\hline & & & & $9.91 \pm 0.09$ \\
\hline \multirow[t]{2}{*}{$\mathbf{F 3}$} & $\beta-[\text { BEDT-TTF }]_{2} \mathrm{I}_{3}$ & $\begin{array}{l}10 \\
20 \\
42 \\
21 \\
24\end{array}$ & $\begin{array}{c}0.25 \\
0.5 \\
1.05 \\
0.5 \\
0.6\end{array}$ & $\begin{array}{c}16.8 \\
18.20 \\
19.30 \\
18.0 \\
18.0\end{array}$ \\
\hline & & & & $18.4 \pm 0.3$ \\
\hline
\end{tabular}

Table S3. Summary of Young's Moduli. Young's Moduli were estimated parallel to sample surface applying uniaxial strain for: F2, F3 and bare PC sample. ${ }^{1}$

\begin{tabular}{ccc}
\hline \hline BL-Film & \multicolumn{1}{c}{ IRS } & Young's Modulus [GPa] \\
\hline \hline F2 & $\alpha-[\text { BEDT-TTF }]_{2} I_{3}$ & $1.90 \pm 0.20$ \\
F3 & $\beta-[\text { BEDT-TTF }]_{2} I_{3}$ & $1.46 \pm 0.20$ \\
PC & None & $2.05 \pm 0.20$ \\
\hline \hline
\end{tabular}




\section{S4) Hydrosensitivity of BL-Films}

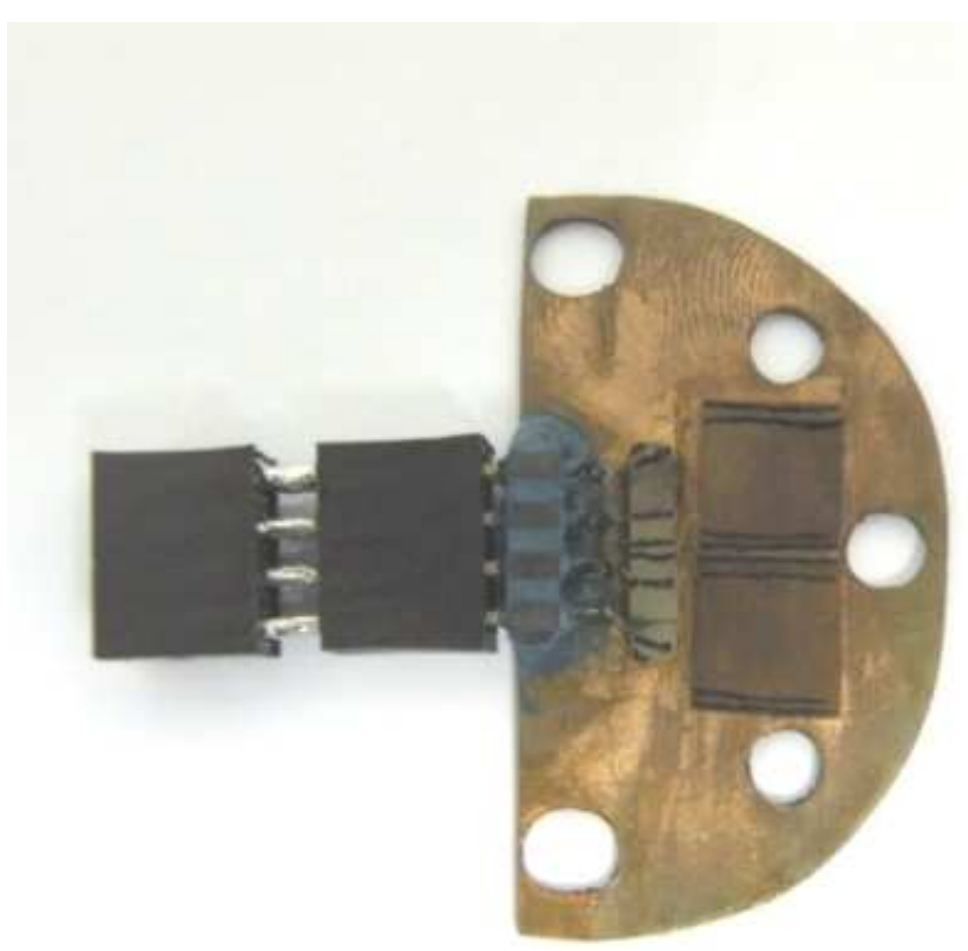

Figure S6. Photo of the homemade copper support with two samples of BL films F1 for simultaneous measurements of the electrical response to relative humidity changes. Copper support was used to guarantee both mechanical and thermal stabilization once the sample was placed within Memmert Oven for humidity experiments. For each sample 4 graphite contacts were used to measure 4-wire dc-resistance. Current of about $1-10 \mu \mathrm{A}$ was applied to prevent Joule heating of the sample. 

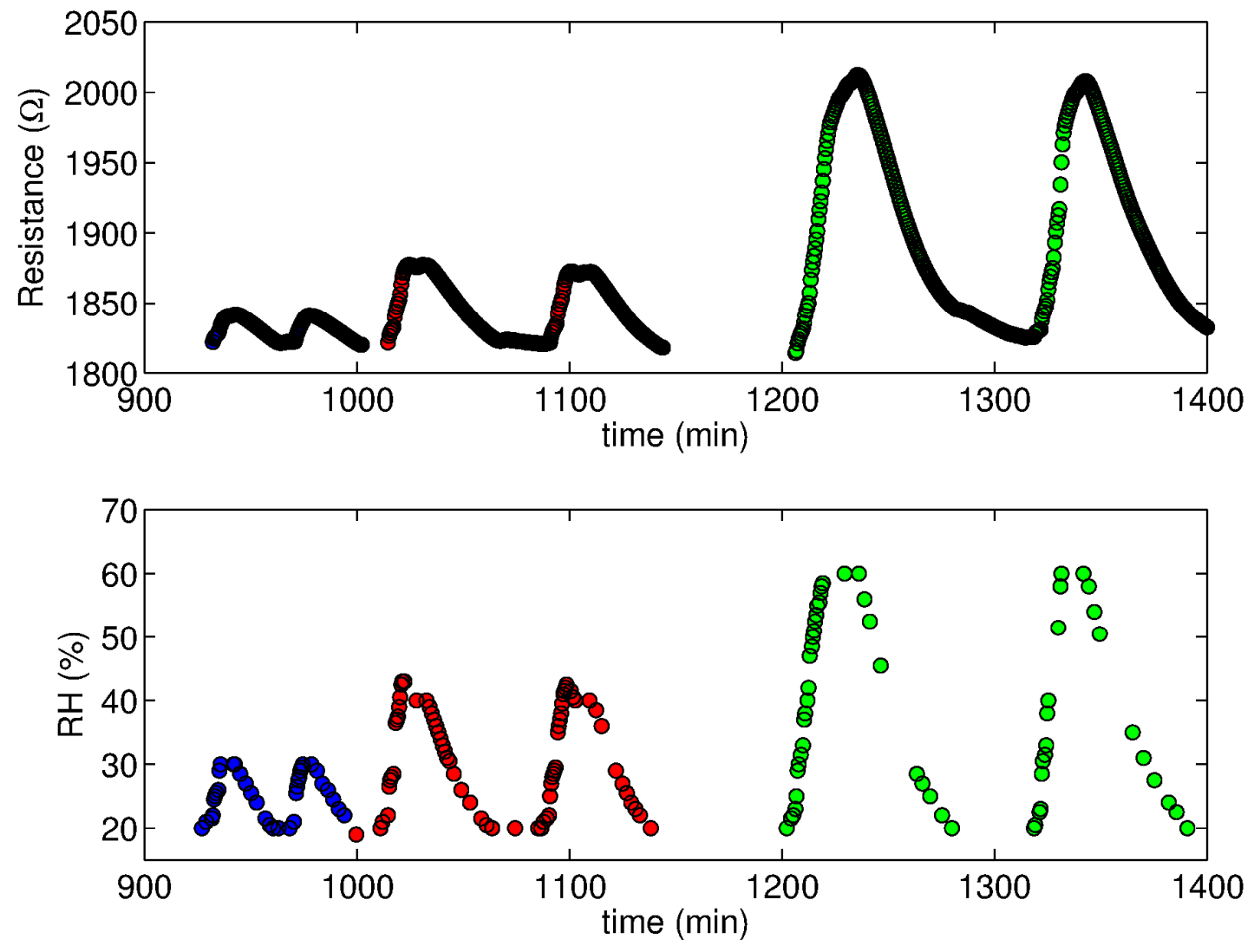

Figure S7. Reversibility of resistance change at different relative humidity. Top) resistance change. Bottom) change of relative humidity (RH). Experiment carried out using samples shown in Figure S6 within a Memmert Oven. Blue, red and green traces correspond to maximum humidity of $30 \%, 40 \%$ and $60 \%$, respectively. 

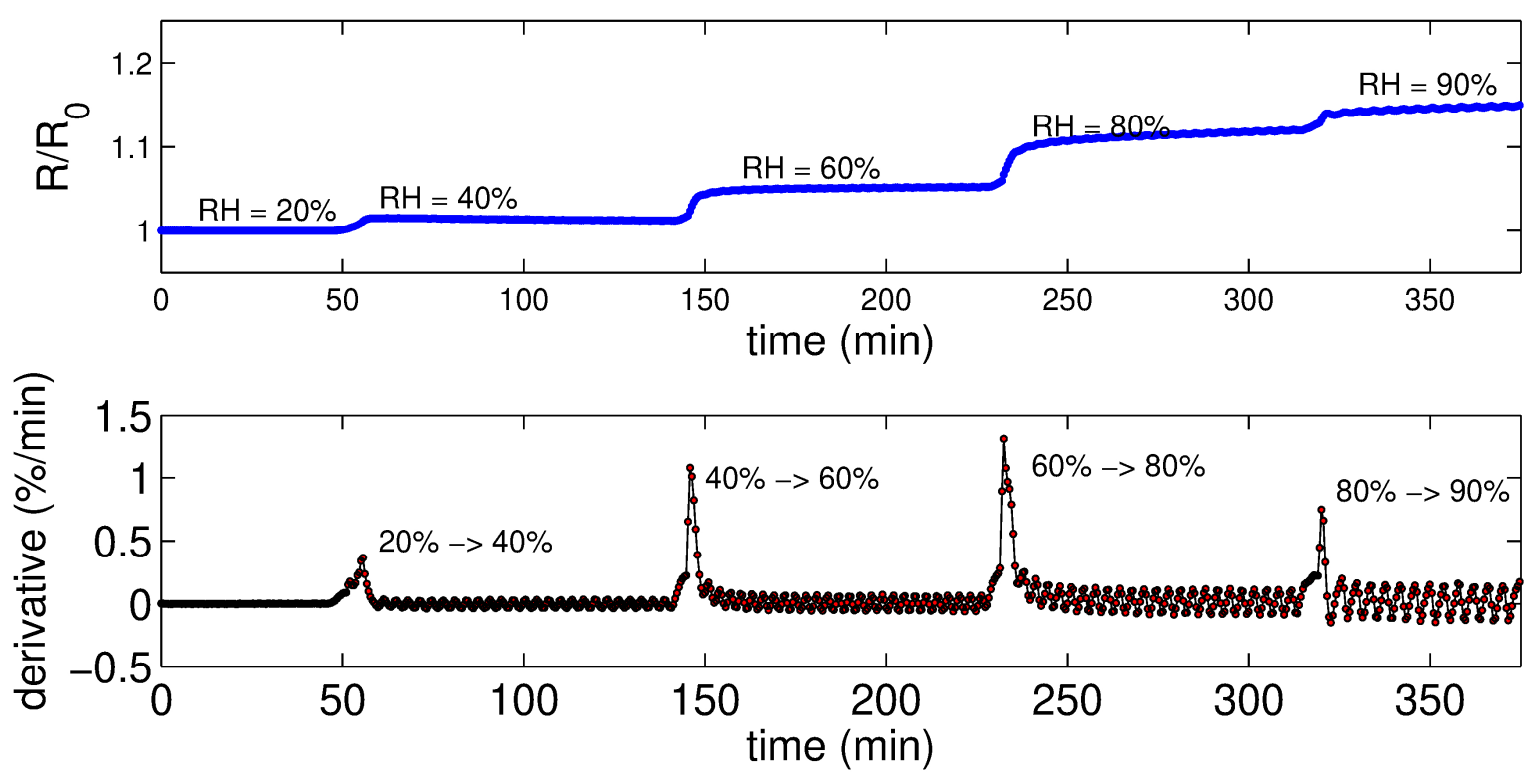

Figure S8. Stability of F1 resistance at different humidity. Top) resistance change and bottom) time derivative of resistance change at different relative humidity $(\phi)$ of samples shown in Figure S6. Measurement carried out within Memmert oven. Stabilization time of resistance after changing humidity level was found to be: $\mathrm{t}=10 \pm 2 \mathrm{~s}$, extracted for humidity steps including: $\Delta \phi=20 \%$ to $40 \%, \Delta \phi=40 \%$ to $60 \%, \Delta \phi=60 \%$ to $80 \%$ and $\Delta \phi=80 \%$ to $90 \%$. Also clearly visible is the increasing step-height of derivative for $\Delta \phi=20 \%$ to $40 \%, \Delta \phi=40 \%$ to $60 \%, \Delta \phi=60 \%$ to $80 \%$. The lower step at $\Delta \phi=80 \%$ to $90 \%$ was attributed to the humidity chamber which exhibits limited control of absolute $\mathrm{RH}$ at $90 \%$, in agreement with the specifications given by the manufacturer. 


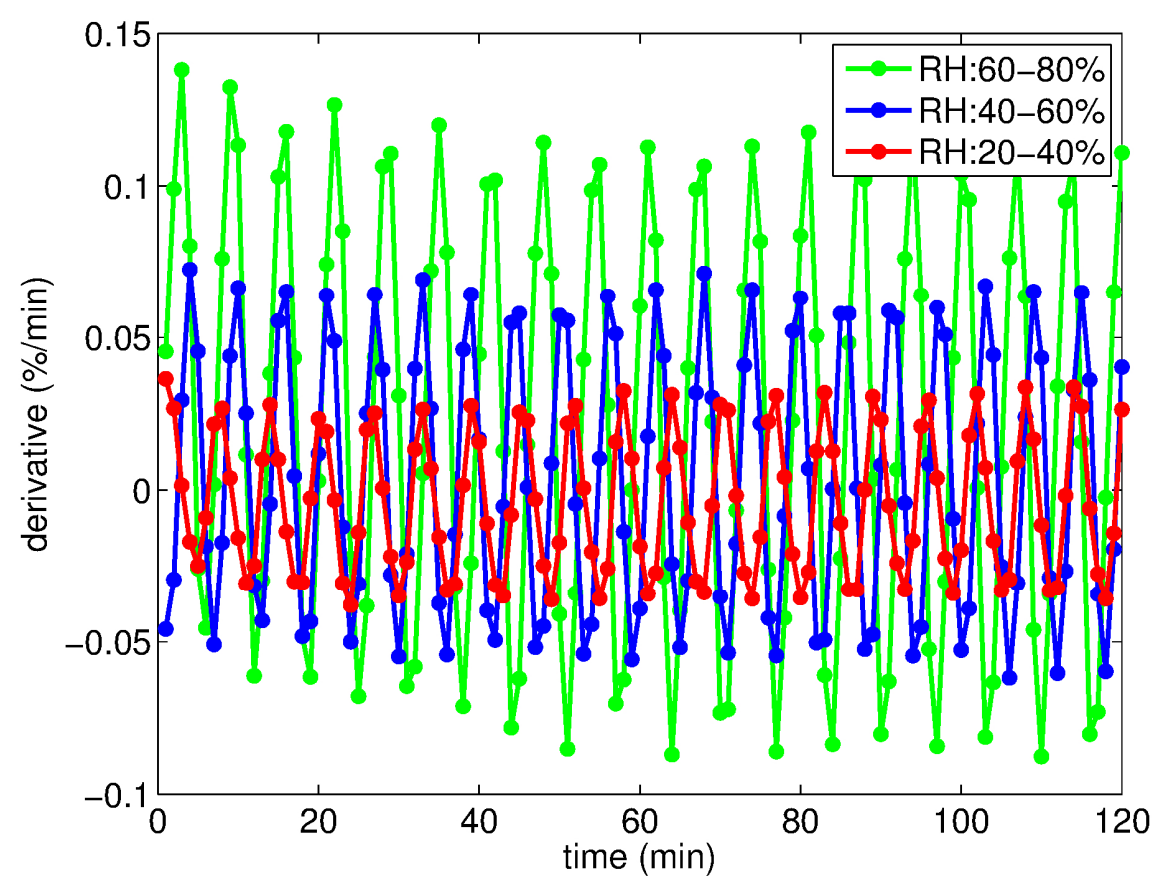

Figure S9. Response speed of F1 resistance to humidity variations. Magnification of derivative in Figure $\mathbf{S 8}$ for three different humidity levels. Ripples in the derivative are due to automatic stabilization of Memmert humidity chamber and well in agreement with manufacturer device specifications. Due to the high humidity sensitivity of BL-Film F1 stabilization cycles of the humidity chamber can be clearly resolved even though the incorporated sensor on top of the chamber showed a constant value of humidity. At higher humidity levels, ripples are bigger, well in agreement with higher humidity sensitivity of BL-Films. 


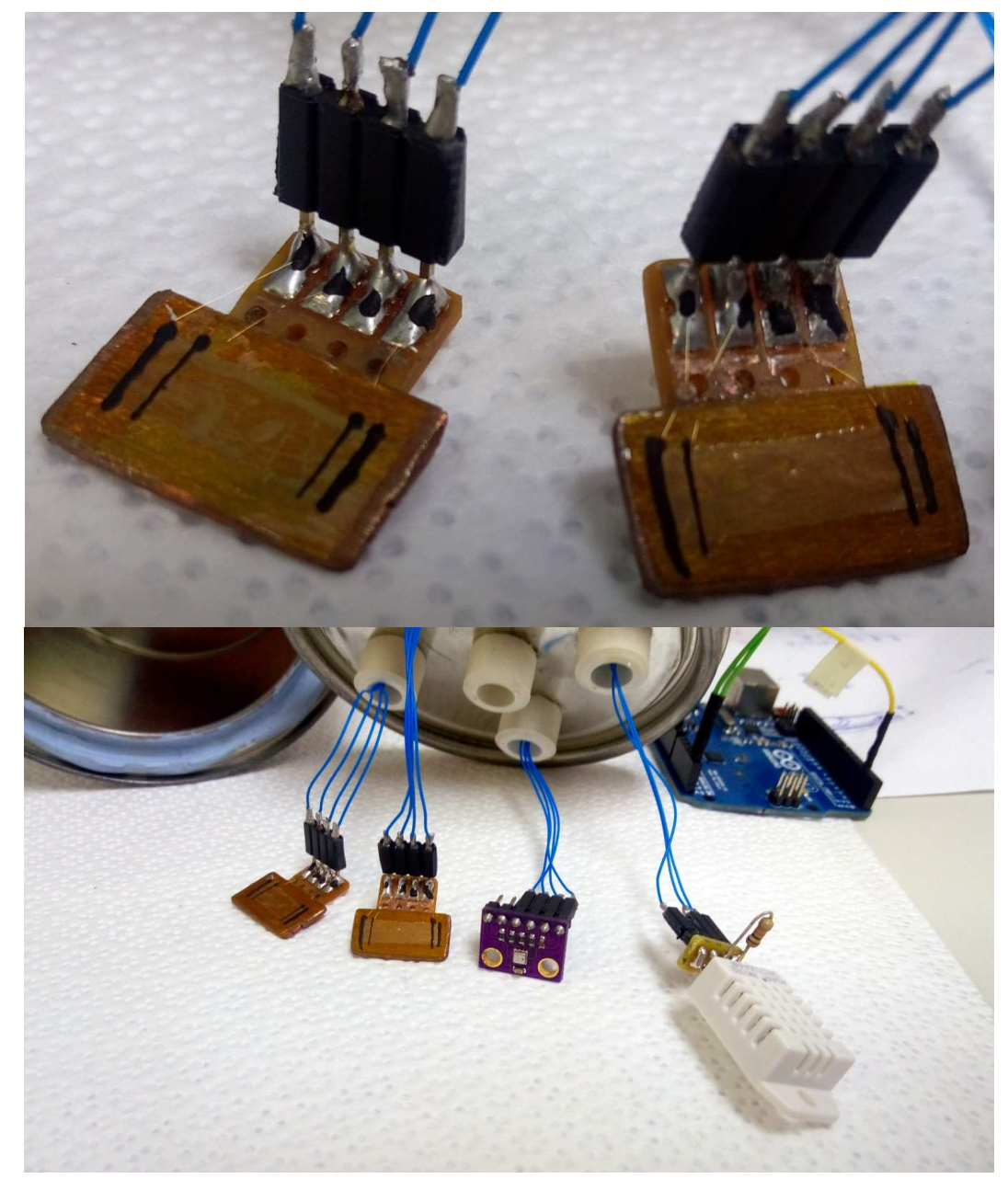

Figure S10. Samples and homemade humidity measurement cell. Commercial sensors (BOSCH BME280 and DHT22) connected to Arduino board have been used to measure temperature, humidity and ambient pressure in real time. Two samples of F1 (F1a and F1b in Figure 2) are mounted on a metal copper substrate for thermal stabilization, electrically insulated by a Kapton layer from the substrate and electrically connected using 4 graphite paste pads and $\mathrm{Au}$ wires for 4-wire dc-resistance measurements. Electrical measurements were carried out using a two channel Keithley 2612 Source Meter with current values of $1-10 \mu$ A to prevent Joule heating of the samples. 

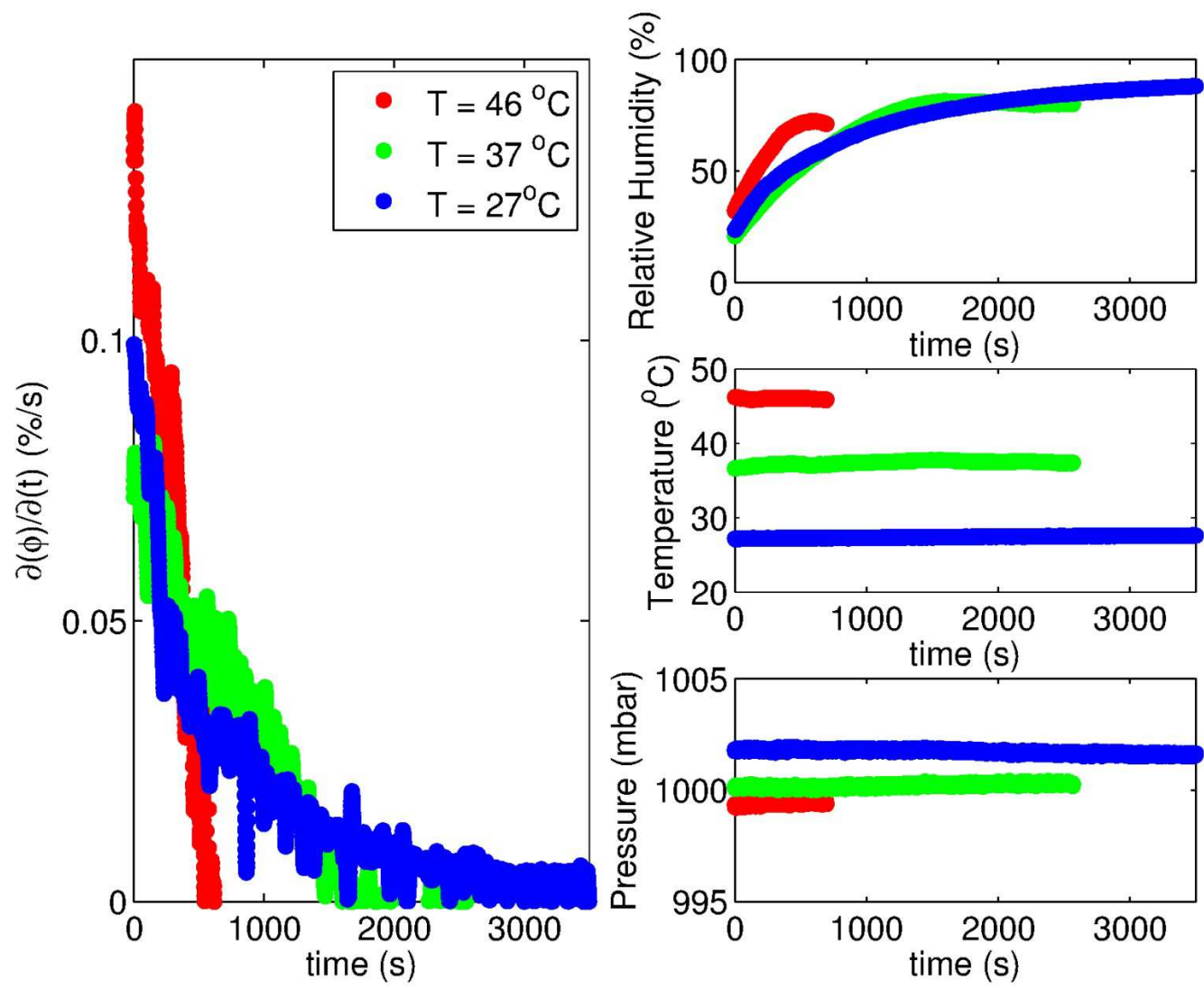

Figure S11. Parameter variation within homemade humidity chamber during resistance measurements. Left) Time-derivative of relative humidity $(\phi)$ corresponding to variation speed of relative humidity was found to be about $0.10 \pm 0.05 \% / \mathrm{s}$, shortly after changing humidity inside the measurement chamber. In order to study solely humidity influence on the resistance change, temperature and ambient pressure inside the measurement chamber have been monitored in real-time during the whole measurement, exhibiting constant values as shown in plots on the right side. 

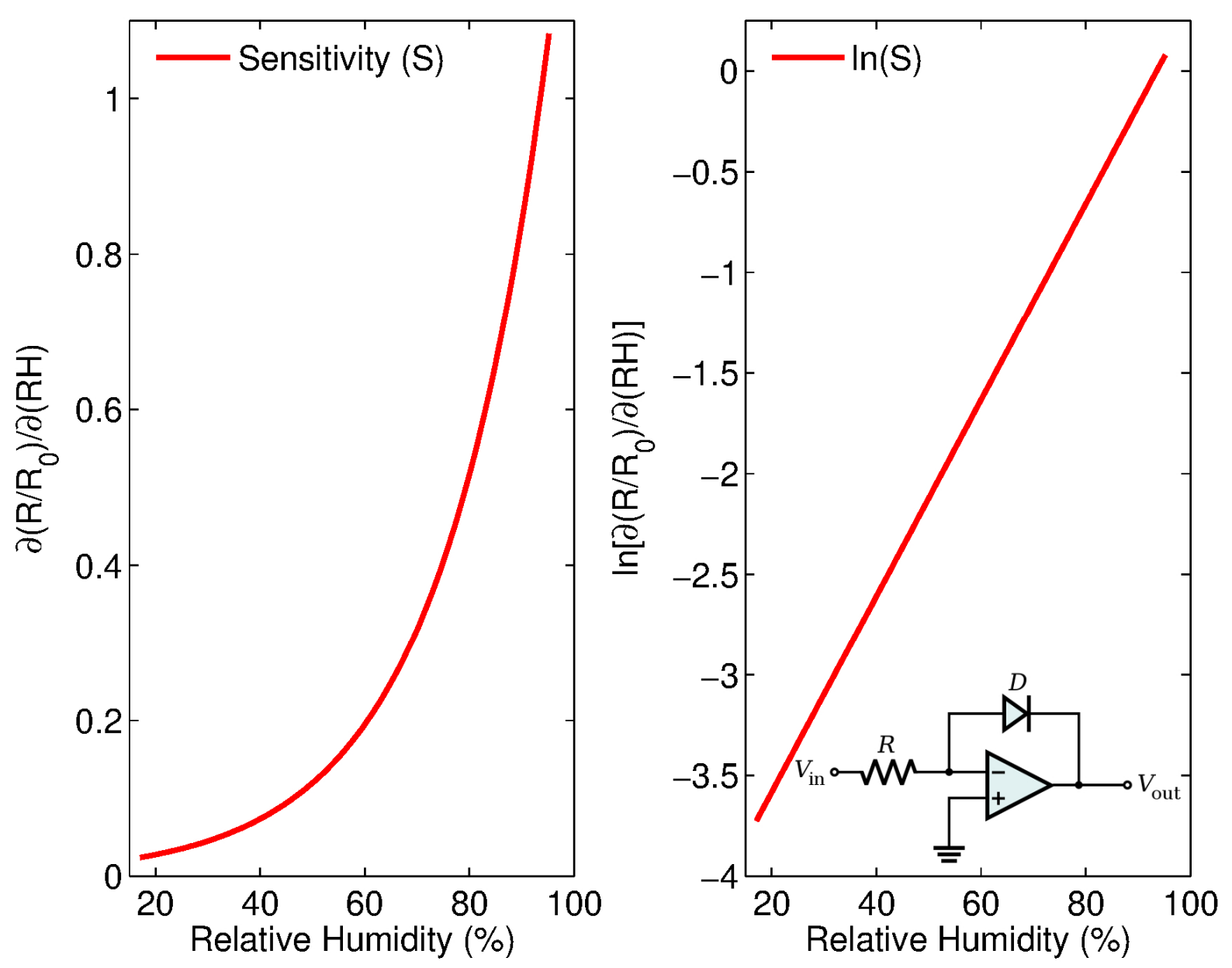

Figure S12. Sensitivity of humidity sensor. Left) Sensitivity for a typical sensor F1 (extracted from fitted curve in Figure 1d) $\partial\left(\mathrm{R} / \mathrm{R}_{0}\right) / \partial(\mathrm{RH})$ as function of relative humidity (RH). Right) Signal linearization after employing a logarithmic amplifier shown in the figure inset. 


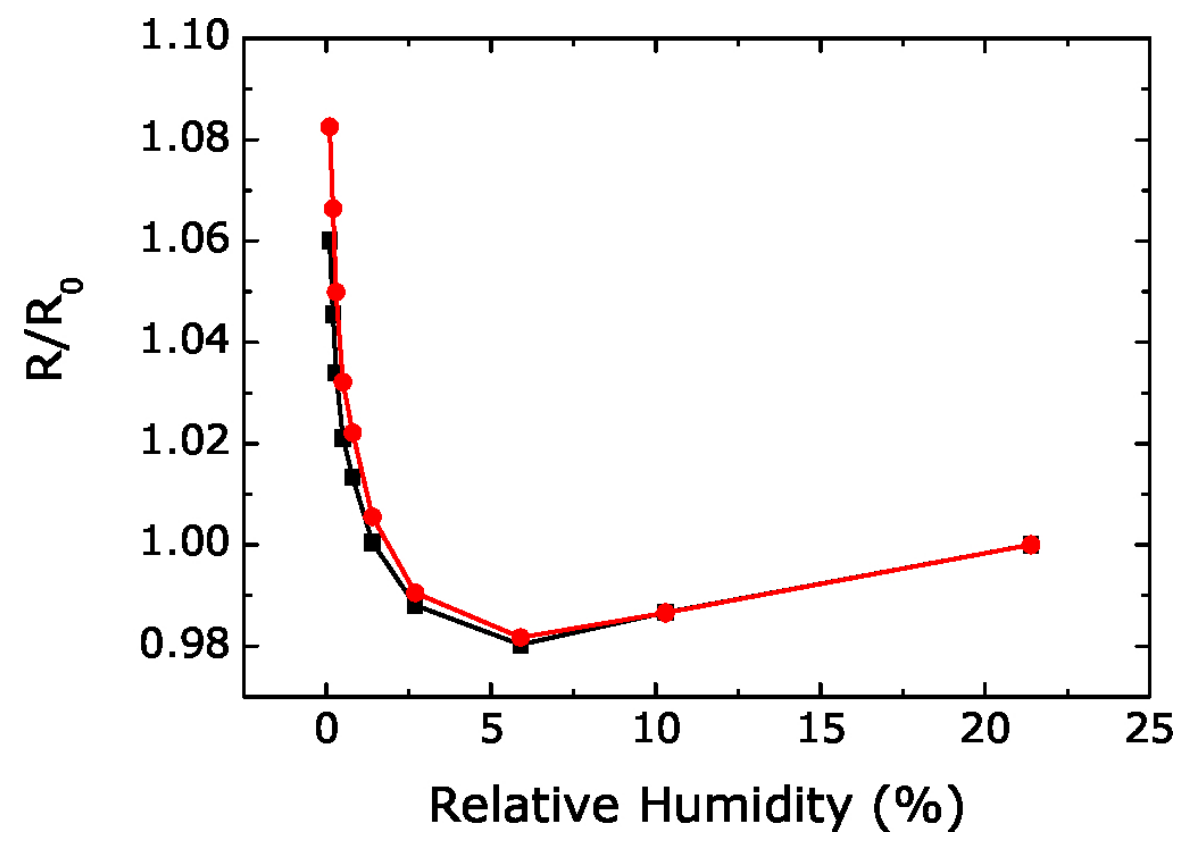

Figure S13. Humidity dependence of normalized electrical resistance $\left(R / R_{0}\right)$ at $T=25{ }^{\circ} \mathrm{C}$ for two representative films F1. Samples were measured within homemade chamber that allowed measuring both relative humidity and electrical resistance. At low relative humidity, i.e. $\mathrm{RH}<5 \%$, a strong increase of the electrical resistance was observed. This might indicate another phase transition under very dry conditions. 


\section{S4.1) Wheatstone bridge and BL-Films for low cost electronics}

The Wheatstone bridge was invented by Samuel Hunter Christie ${ }^{6}$ in 1833 and improved and popularized by Sir Charles Wheatstone ${ }^{7}$ in 1843 . This configuration is often used in sensor technology and permits to decouple multidimensional signal variations of sensor elements. It can be used to eliminate the absolute value, reducing the measurement scale and thereby simplifying data acquisition permitting increased resolution of the measurement. It is typically formed by four branches consisting of active $\left(R_{\text {sensor }}\right)$ and passive $\left(R_{\text {reference }}\right)$ sensor elements and a variable resistor $\left(\mathrm{R}_{\text {variable }}\right)$ to balance the bridge (i.e. absolute offset removal). Two possible scenarios are depicted in Figures S14a and S14b, with one and two active sensors, respectively. Once the active sensor (or sensors) is/are stimulated a voltage signal proportional the sensor response can be measured by the Analog Digital Converter (ADC).

Similar structures have been used previously in BL-film based sensors in contactlens (to measure the intraocular pressure of a human eye) and in contact less radiation sensors

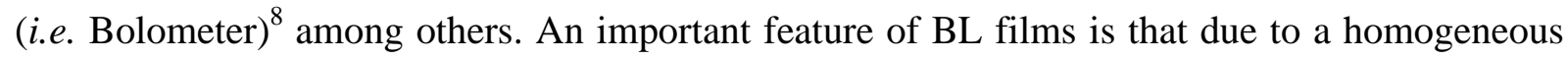
active layer thickness of the polycrystalline topmost layer, square pieces of the same sample exhibit the same sheet resistance $\left(\mathrm{R}_{\square}\right)$ even if the absolute area is different (see Figure S14c). This permits to use the same film as active (directly exposed to stimulus, $R_{\text {sensor }}$ ) and passive (protected from stimulus i.e. through its encapsulation, $\mathrm{R}_{\text {reference }}$ ) sensor element. More interestingly, for RH humidity sensors, it also allows to use for instance F1 (highly sensitive to RH changes) as active sensor and $\mathbf{F 3}$ (not sensitive to RH changes) as passive sensor.

Additionally, resistance, current and voltage ranges of such sensor elements are fully compatible with emerging low cost open-source electronic systems, shown schematically in Figure S14. $^{9}$ 

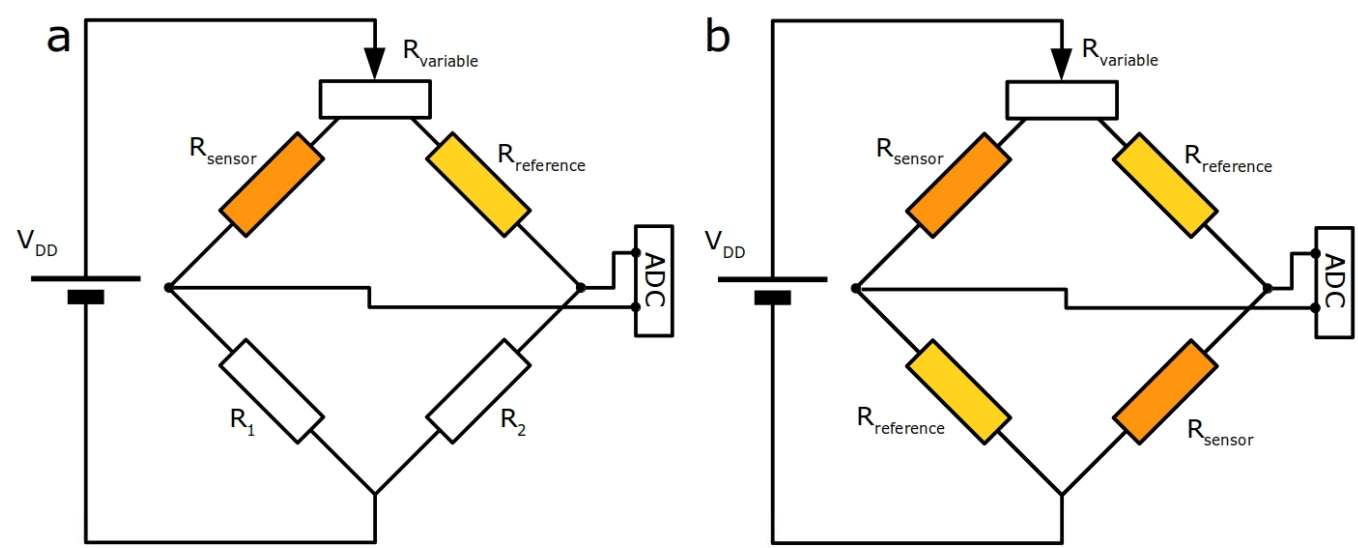

C

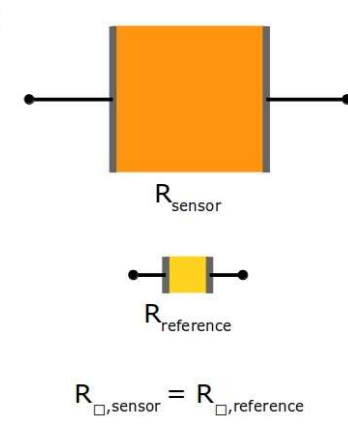

d

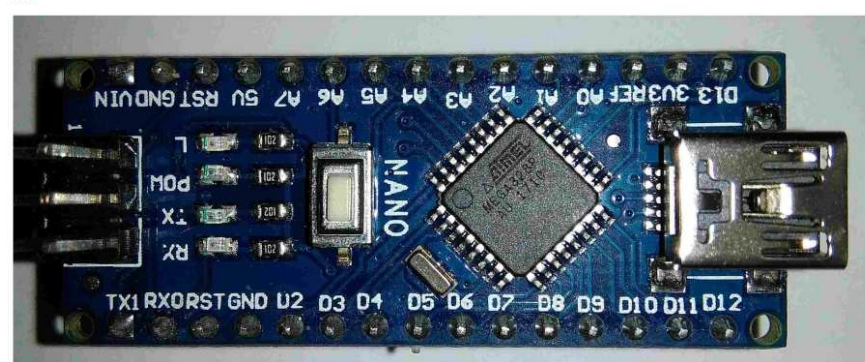

Figure S14: BL-films connected in a Wheatstone bridge shown schematically. Bridges consisting of one active sensor $\left(\mathrm{R}_{\text {sensor }}\right)$ (a) and two active sensors $\left(\mathrm{R}_{\text {sensor }}\right)(\mathrm{b})$, respectively Variable resistor $\left(\mathrm{R}_{\text {variable }}\right)$ is used to electrically balance the bridge. Reference sensor $\left(\mathrm{R}_{\text {reference }}\right)$ is used to decouple multidimensional sensor response. (Response measured at Analog Digital converter (ADC) for (b) is 2 times bigger compared to (a)). c) Square pieces of the same sample exhibit the same sheet resistance $\left(R_{\square}\right)$ even though absolute area is different. $\left.d\right)$ Low cost open source board, Arduino nano, fully compatible with BL-films (i.e. $\mathrm{V}_{\mathrm{DD}}=5 \mathrm{~V}$ ). Arduino Nano taken from (https://commons.wikimedia.org/wiki/File:Arduino_Nano.jpg; David Mellis [CC BY 2.0 (https://creativecommons.org/licenses/by/2.0)], No modifications were done to the image. 


\section{S5) Boltzmann's sigmoidal equation and de-convolution of relative resistance change}

The mathematical model proposed by Boltzmann in 1879 was based on the sigmoidal logistic equation: $f(x)=1 /\left[1+e^{x}\right]$. This equation has been used to describe behaviors exhibited when a certain factor triggers a transition (reversible or not) from a steady state to another one with very different magnitude. This is an appropriate starting point for the prediction of transition phenomena. Thus, the following Boltzmann's sigmoidal equation, where the original function was modified, which contains the required geometric characteristics, is proposed to describe changes in d-spacing observed for $\mathbf{F 1}$ :

$$
f(x)=a+\frac{b-a}{1+e^{\frac{x-x_{0}}{c}}}
$$

Equation (1)

where $\mathrm{a}$ and $\mathrm{b}$ are the equilibrium values of the dependent variable before and after the transition, respectively; $\mathrm{x}_{0}$ is an inflection point and $\mathrm{c}$ is a coefficient that describes the behavior of the slope of the process during the transition. Figure S15 show the extracted relative change of d-spacing $\left(\Delta \mathrm{d} / \mathrm{d}_{0}\right)$ increasing $\mathrm{RH}$ levels in the range from $10 \%$ to $80 \%$ with its fit employing equation 1. Geometrical parameters, as well as goodness of fit $\left(\mathrm{R}^{2}\right)$ are reported in the inset. Figure S16 shows the corresponding fit for decreasing RH. 


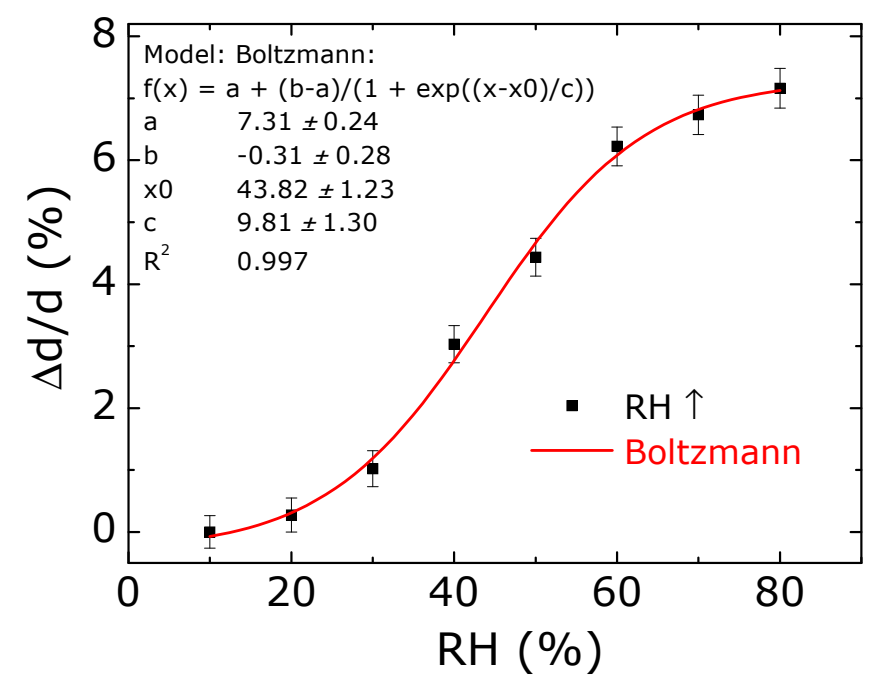

Figure S15: Relative humidity dependence of relative d-spacing. Boltzmann's sigmoidal function used to model $\Delta \mathrm{d} / \mathrm{d}_{0}$ for increasing $\mathrm{RH}$ levels in the range from $10 \%$ to $80 \%$. Geometrical parameters and goodness of fit $\left(\mathrm{R}^{2}\right)$ reported in the inset. Error bars correspond to standard errors obtained in Gaussian peak deconvolution.

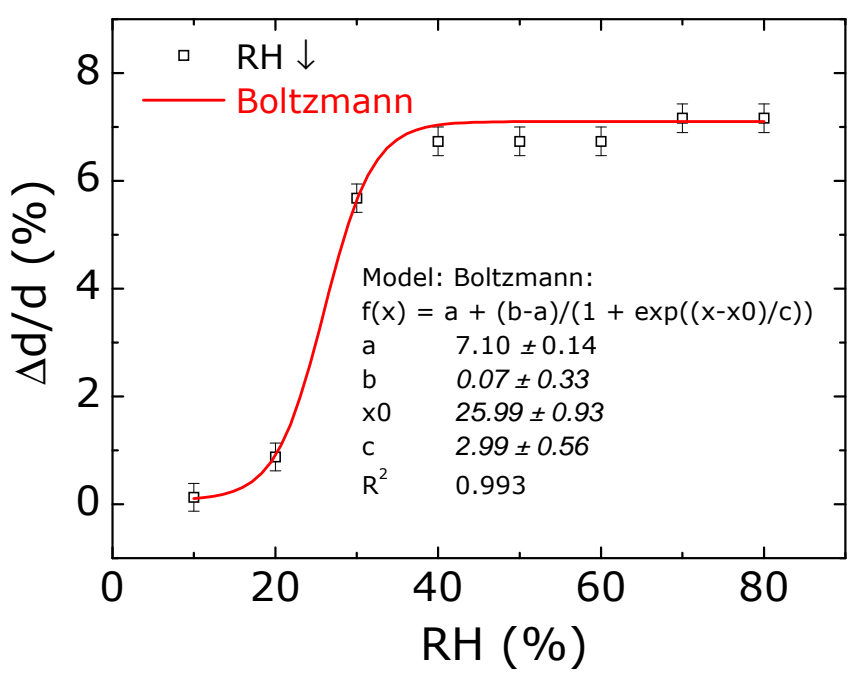

Figure S16: Relative humidity dependence of relative d-spacing. Boltzmann's sigmoidal function used to model $\Delta \mathrm{d} / \mathrm{d}_{0}$ for decreasing $\mathrm{RH}$ levels in the range from $80 \%$ to $10 \%$. Geometrical parameters and goodness of fit $\left(\mathrm{R}^{2}\right)$ reported in the inset. Error bars correspond to standard errors obtained in Gaussian peak deconvolution. 
Figure S17 shows the $1^{\text {st }}$ derivatives of $\Delta \mathrm{d} / \mathrm{d}_{0}$ with respect to $\mathrm{RH}$ reported in Figures $\mathbf{S 1 5}$ and S16. Derivative calculated for increasing RH exhibited a slope of $0.19 \pm 0.01(\% / \% \mathrm{RH})$ at a $\mathrm{RH}$ of about $44 \pm 1 \%$, while derivative calculated for decreasing RH exhibited a slope of $0.59 \pm$ $0.01(\% / \% \mathrm{RH})$ at a $\mathrm{RH}$ of about $26 \pm 1 \%$. This leads to a total hysteresis in $\mathrm{RH}$ of about $18 \%$.

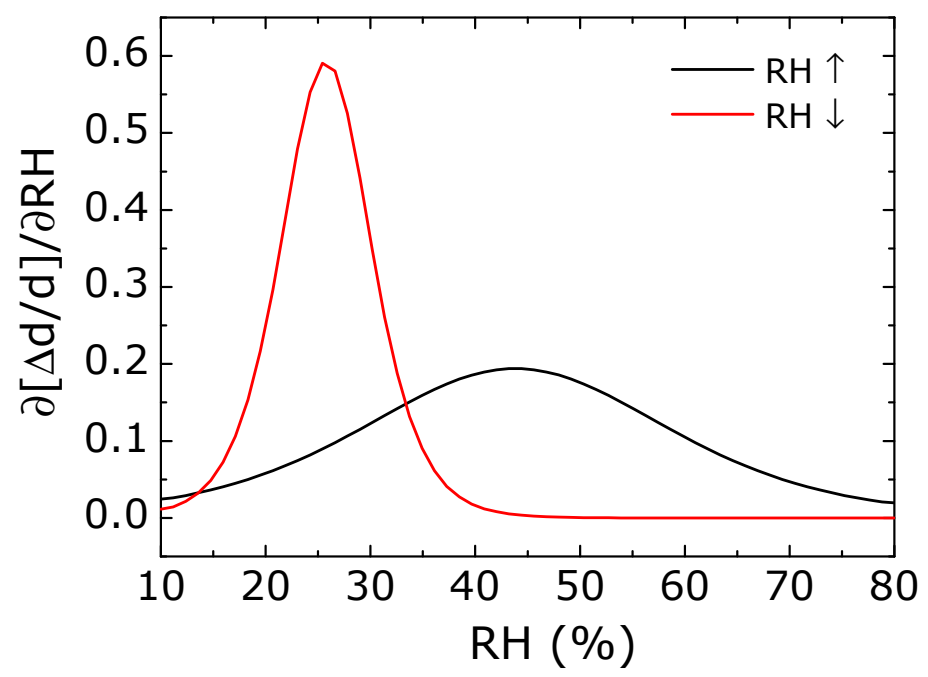

Figure S17: Derivative of relative d-spacing over RH. Calculated maximum values correspond to slopes in Figures S13 and S14 exhibiting $0.19 \pm 0.01 \% / \%$ RH and $0.59 \pm 0.01$ $\% / \% \mathrm{RH}$ for increasing and decreasing $\mathrm{RH}$, respectively.

Figure $\mathrm{S18}$ shows the relative resistance change $\left(\mathrm{R} / \mathrm{R}_{0}\right)$ as function of relative d-spacing change $\left(\Delta d / d_{0}\right)$. Clearly two regimes have been identified and described by the following expression:

$$
R=R_{0}\left[100+a \cdot x+b \cdot\left(x-x_{c}\right)\right]^{t}
$$

Equation (2)

Figure S19 shows the deconvolution exhibiting $\mathrm{a}=0.38 \pm 0.01\left(\right.$ in $\left.\% / \% \Delta \mathrm{d} / \mathrm{d}_{0}\right), \mathrm{x}_{\mathrm{c}}=7.3$ $\pm 0.2\left(\right.$ in $\left.\% \Delta \mathrm{d} / \mathrm{d}_{0}\right), \mathrm{b}=1$ and $\mathrm{t}=-1$. 


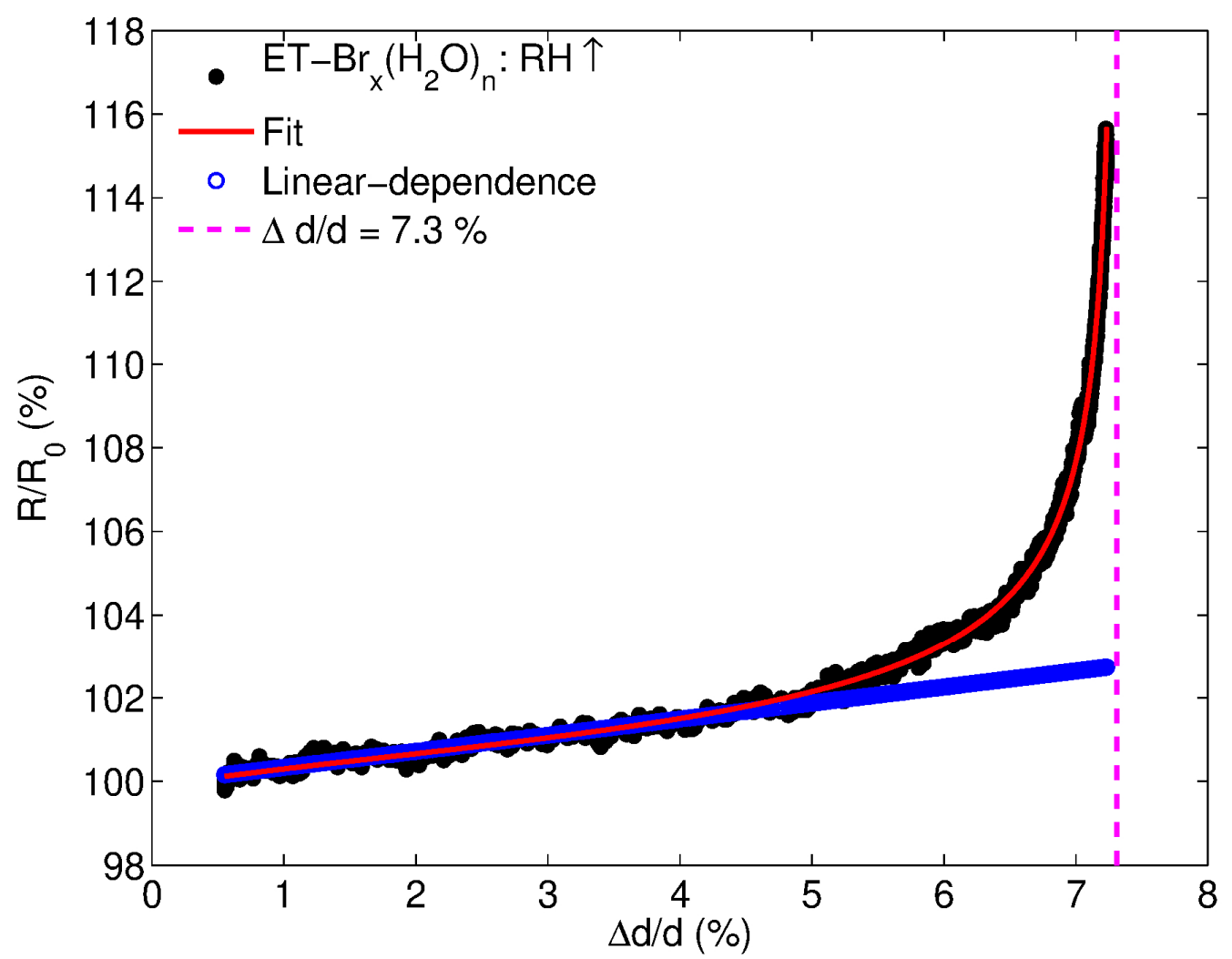

Figure S18: Relative resistance change $\left(R / R_{0}\right)$ versus relative $d$-spacing $\left(\Delta d / d_{0}\right)$ for $F 1$ and increasing RH. Two regimes have been found: i) linear $\mathrm{R} / \mathrm{R}_{0} \Delta \mathrm{d} / \mathrm{d}_{0}$ dependence for $\Delta \mathrm{d} / \mathrm{d}_{0}<4.5$ $\%$ and ii) strongly increasing $\mathrm{R} / \mathrm{R}_{0}$ for $\Delta \mathrm{d} / \mathrm{d}_{0}>4.5$. Critical value of $\Delta \mathrm{d} / \mathrm{d}_{0}=7.3 \pm 0.2 \%$ is in agreement with parameter a fitted in Figure S15. 


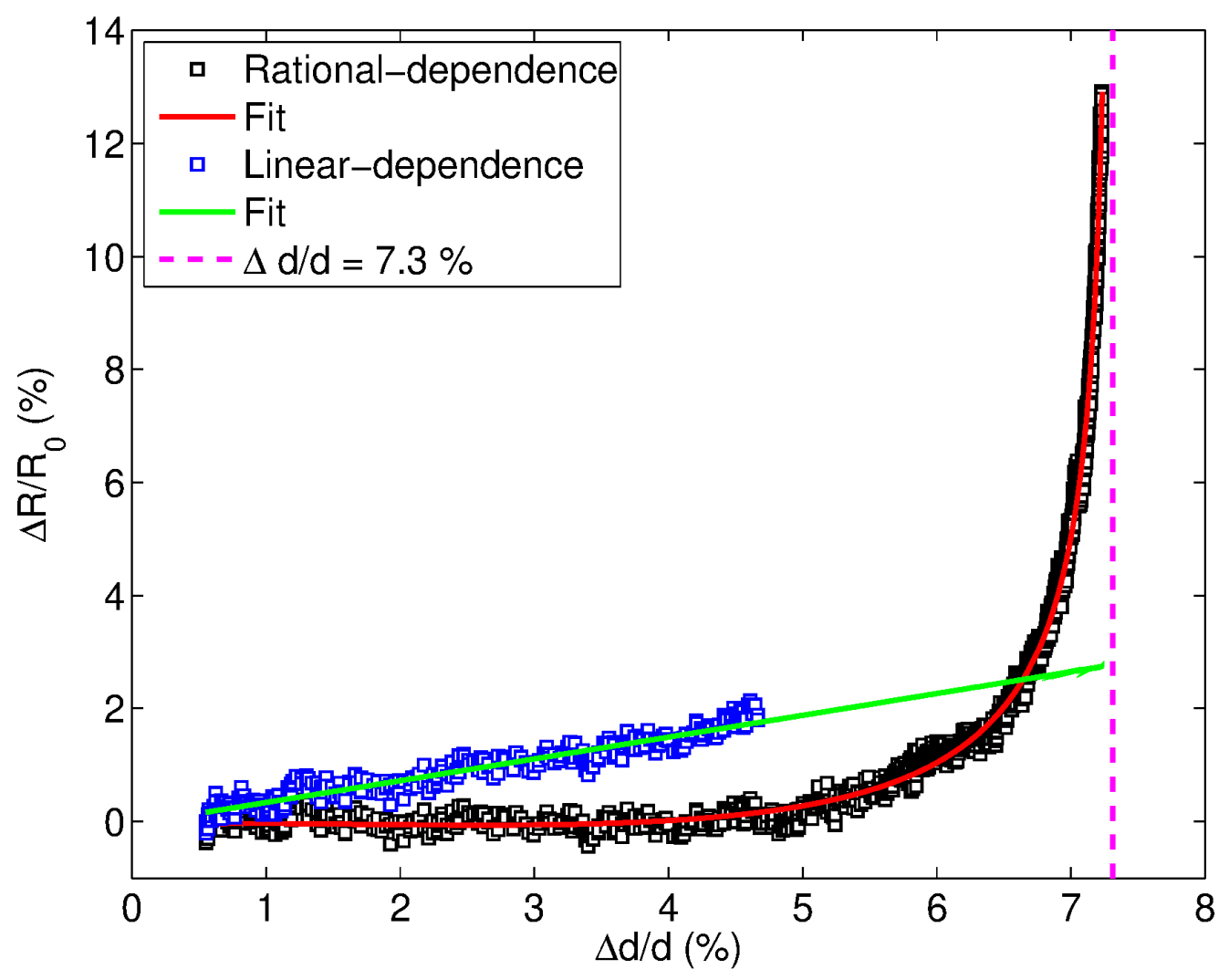

Figure S19: Deconvolution of relative resistance change $\left(R / R_{0}\right)$ versus relative $d$-spacing $\left(\Delta \mathbf{d} / \mathbf{d}_{0}\right)$ for $\mathbf{F 1}$ and increasing $\mathbf{R H}$. Two regimes have been found: i) linear dependence $R / R_{0}$ $\Delta \mathrm{d} / \mathrm{d}_{0}$ dependence for $\Delta \mathrm{d} / \mathrm{d}_{0}<4.5 \%$ and ii) strongly increasing $\mathrm{R} / \mathrm{R}_{0}$ (rational dependence: $\mathrm{R} / \mathrm{R}_{0}$ $\left.\approx \mathrm{b} \cdot\left(\mathrm{x}_{\mathrm{c}}-\mathrm{x}\right)^{\mathrm{t}}\right)$ with critical value of $\mathrm{x}_{\mathrm{c}}=\Delta \mathrm{d} / \mathrm{d}_{0}=7.3 \pm 0.2 \%$ and critical exponent $\mathrm{t}=-1$, is in agreement with parameter a fitted in Figure $\mathbf{S 1 5}$. 


\section{REFERENCES}

(1) Lebedev, V. PhD. Thesis: Towards Plastic Electronics Sensing Devices With TTF-based Molecular Conductors as Active Components. Autonomous University of Barcelona, ICMAB-CSIC, 2014.

(2) Brooks, A. C.; Martin, L.; Day, P.; Lopes, E. B.; Almeida, M.; Kikuchi, K.; Fujita, W.; Sasamori, K.; Aktusu, H.; Wallis, J. D. Hydrogen bonded anion ribbons, networks and clusters and sulfur-anion interactions in novel radical cation salts of BEDT-TTF with sulfamate, pentaborate and bromide. Dalton Transactions 2013, 42 (18), 6645-6654.

(3) Zhang, Q.; Wu, P.; Li, Y.; Zhu, D. Synthesis, structure and physical properties of ET2Br·3H2O. Synthetic Metals 1998, 98 (2), 129-133.

(4) Kobayashi, H.; Kobayashi, A.; Sasaki, Y.; Saito, G.; Inokuchi, H. The Crystal and Molecular Structures of Bis(ethylenedithio)tetrathiafulvalene. Bulletin of the Chemical Society of Japan 1986, 59 (1), 301-302.

(5) Laukhina, E.; Pfattner, R.; Ferreras Lourdes, R.; Galli, S.; Mas-Torrent, M.; Masciocchi, N.; Laukhin, V.; Rovira, C.; Veciana, J. Ultrasensitive Piezoresistive All-Organic Flexible Thin Films. Advanced Materials 2009, 22 (9), 977-981.

(6) Christie Samuel, H. VIII. The Bakerian Lecture.-Experimental of laws of magneto-electric induction in different masses of the same metal, and of Intensity in different metals. Philosophical Transactions of the Royal Society of London 1833, 123, 95-142.

(7) Wheatstone, C. XIII. The Bakerian lecture.-An account of several new instruments and processes for determining the constants of a voltaic circuit. Philosophical Transactions of the Royal Society of London 1843, 133, 303-327.

(8) Pfattner, R.; Lebedev, V.; Laukhina, E.; Kumar Suddapalli, C.; Esteban-Martin, A.; Ramaiah-Badarla, V.; Ebrahim-Zadeh, M.; de Arquer Pelayo García, F.; Konstantatos, G.; Laukhin, V.; Rovira, C.; Veciana, J. A Highly Sensitive Pyroresistive All-Organic Infrared Bolometer. Advanced Electronic Materials 2015, 1 (8), 1500090.

(9) Arduino Project started at Interaction Design Institute Ivrea (IDII) in Ivrea, Italy. https://www.arduino.cc/. 